\title{
Calibration, performances and tests of the first detection unit of the KM3NeT neutrino telescope
}

\author{
Creusot Alexandre* for the KM3NeT collaboration \\ Laboratoire APC, Paris \\ E-mail: creusoteapc.in2p3.fr
}

\begin{abstract}
$\mathrm{KM} 3 \mathrm{NeT}$ is the next generation neutrino telescope being installed in the Mediterranean Sea. The first detection unit of the telescope is ready for installation in the deep Mediterranean Sea in the summer of 2015. Eighteen digital optical modules have been mounted on a vertical string for the detection of the Cherenkov light emitted by muons induced by up-going neutrinos. This paper reports on the integration and calibration of the optical modules and of the full detection unit, as well as the future installation in the deep sea and the on-shore operation. The additional information gained by the new type of photo-detection units when comparing to the old generation of optical modules is also discussed.
\end{abstract}

The 34th International Cosmic Ray Conference,

30 July- 6 August, 2015

The Hague, The Netherlands

${ }^{*}$ Speaker. 


\section{Introduction}

The KM3NeT Observatory [1] is a large scale neutrino telescope to be deployed in the deep Mediterranean sea. The Italian site (KM3NeT-IT) off Capo-Passero shore, Sicily, will be dedicated to the ARCA project (Astrophysics Research with Cosmics in the Abyss), i.e. the detection of high energy astrophysics neutrinos. The French site (KM3NeT-FR) off La Seyne sur mer shore will be dedicated to the ORCA project (Oscillation Research with Cosmics in the Abyss), i.e. the detection of low energy neutrinos for the mass hierarchy study.

With several cubic kilometers of effective volume and several thousands of optical detectors, ARCA will be the largest and most sensitive neutrino detector in the energy range of $1-10000 \mathrm{TeV}$. Being situated in the Northern Hemisphere it will be particularly suited to the investigation of high energy neutrinos from our Galaxy. The signal of neutrinos with energies up to $2 \mathrm{PeV}$ reported recently by the Ice-Cube collaboration [2] has strengthened the motivation for the construction of ARCA. One of the main motivations is therefore to provide a survey of the Universe at large distance and energy scales. For ORCA, the Observatory layout has been designed in order to be sensitive to much lower energies, typically at about $10 \mathrm{GeV}$ [3].

For both the Italian and the French sites, the neutrino detection is based on a sampling of the Cherenkov light emitted by the products of the neutrino interaction in the detector vicinity. The sampling is performed with the Digital Optical Modules (DOM), the elementary units of the KM3NeT arrays. DOMs are structured in vertical lines (18 DOMs per line) which are configured in building blocks of 118 lines. The spacing between DOMs and lines determines the energy range of interest and as a consequence the domain of Physics: few meters for ORCA, several tens of meters for ARCA.

Several DOM prototypes have been deployed in the deep sea the last 2 years. The excellent results $[4,5]$ obtained with all the devices have allowed the validation of the DOM as a multiphotomultiplier tubes (PMT) detector for a large production. The first full vertical line of DOMs, i.e. detection unit 1 (DU1), has been integrated and tested in laboratory. The DU1 is ready for deployment in summer 2015.

The first phase of the KM3NeT telescope is being implemented with the installation of a seafloor and shore infrastructure at the KM3NeT-FR and KM3NeT-IT sites and the construction and installation of 31 detection units (24 in KM3NeT-IT and 7 in KM3NeT-FR). The first phase of construction will be completed in 2016 with an instrumented volume about three times that of ANTARES [6]. The next step will be the completion of the detector volume to that of two building blocks for a total volume 1 to 1.6 times that of IceCube, depending on the spacing of the detection units. This phase is meant for measuring the IceCube high-energy signal. The final step will be the completion of six building blocks forming together a detector with a total instrumented volume of about 3 to $5 \mathrm{~km}^{3}$, depending on the spacing of the detection units between 90 and $120 \mathrm{~m}$.

\section{Detector}

\subsection{Description and status}

The KM3NeT Observatory is based on the concept of all data to shore, meaning that all DOM data are gathered locally at the DU base, then routed to shore through a Main Electro Optical Cable 
(MEOC) and a sub-sea network of cables and junction boxes. For the French infrastructure, the MEOC and the main junction box have been deployed successfully end of 2014 and in spring 2015, respectively. In Capo Passero, the MEOC, the main node and KM3NeT prototypes are successfully operated since 2007; a major refurbishment of the infrastructure, aimed at the installation of 12 DUs is scheduled in July 2015. The next step in the Observatory construction is the DU1 deployment and connection to the main junction box, which should take place during summer 2015.

The DU1 has been assembled in the ARCA configuration: 18 DOMs with a vertical spacing of $36 \mathrm{~m}$. The line is composed of 2 Dyneema ${ }^{\circledR}$ ropes for the structure rigidity, a base container for the power supply and DOM optical data multiplexing and a Vertical Electro Optical Cable (VEOC) for the backbone connections between the DOMs, the base container and the foot of the line. The line is anchored to the sea bed and is kept vertical by a buoy at the upper end. The positioning is constantly monitored via an acoustic system [7]. A sketch of the DU1 is shown in the left plot of figure 1. The foot of the line is connected to the main junction box via an inter-node link. The junction box is connected to the shore station via the MEOC. The line deployment at distances between $40 \mathrm{~km}$ and $100 \mathrm{~km}$ from shore and at depths between $2500 \mathrm{~m}$ and $4500 \mathrm{~m}$ is performed using dedicated vessels and ROVs [8].
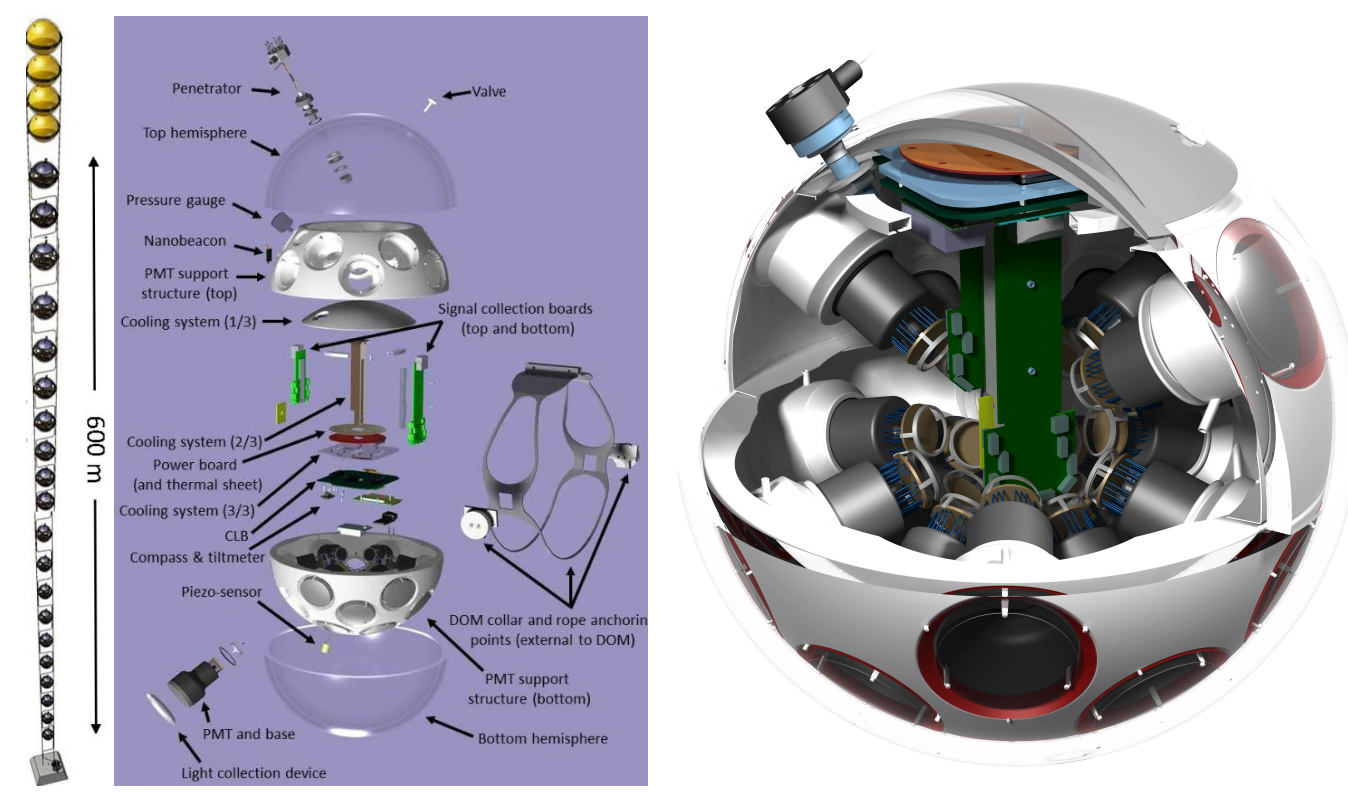

Figure 1: In the left, sketch of the DU1. The 18 DOMs are drawn as well as the top buoy and the anchor. In the middle, exploded view of the DOM, the different components are detailed. In the right, representation of the DOM after assembly.

\subsection{DOMs}

The DOM [9] is a 17" diameter pressure resistant glass sphere comprising 31 PMTs, a piezo for acoustic positioning, an AHRS Compass-and-tilt board for orientation and a nanobeacon for time calibration. The DOM also contains the data acquisition electronics, the communication modules, the power supply board and an SFP laser transceiver for long range communication to shore.

The PMT signals are digitized by a custom designed active base. A comparator is used to produce 
an LVDS signal when the PMT signal amplitude exceeds a threshold of 0.3 spe. The threshold can be adjusted from shore. The LVDS signal is then processed by TDCs implemented in the Central Logic Boards (CLB). Three items are extracted for each signal, the arrival time referring to a common clock, the signal Time over Threshold (ToT) characteristic of the signal amplitude, and the PMT identity. The timing accuracy is below $1 \mathrm{~ns}$. The main characteristics of the photomultipliers used for DU1 (Hamamatsu R12199-02) are a maximum quantum efficiency of 30\%, a transition time spread (TTS) below $5 \mathrm{~ns}$, a high gain of about $5 \cdot 10^{6}$, a dark current rate below $500 \mathrm{~Hz}$ and an after-pulses contamination below $10 \%$. Each sensor module dissipates about $7 \mathrm{~W}$. An exploded view and a drawing of the DOM are shown in the center and right plots of figure 1, respectively. It has been shown in [4] that the photocathode segmentation into 31 pieces allows an efficient rejection of the background produced by the bioluminescence and by the decay of the ${ }^{40} \mathrm{~K}$ contained in sea water. Even with a single DOM, the identification of atmospheric muon signals could be demonstrated allowing an estimate of the muon flux at the respective depth.

The nanobeacon is a pulsed LED light-source for use as a multi-purposes calibration device. Its rise-time is about $2 \mathrm{~ns}$ and its FWHM about $5 \mathrm{~ns}$. The wavelength of the photons is in the range $[350 ; 550 \mathrm{~nm}]$. More than $10^{8}$ photons are emitted per pulse in an opening angle of $5^{\circ}$.

\subsection{Data acquisition system}

The crucial points in the all data to shore strategy are the time tagging of the PMT hits and their transfer to the shore station. For this purpose, a synchronized Ethernet network is provided between all DOMs located in the deep sea and the shore station. The desired sub-nanosecond accuracy and precision of this network are accomplished by adapting the White Rabbit (WR) technology to the KM3NeT requirements [10]. Once the hits are time tagged offshore by the CLB, they are transferred to the shore station in discrete time slices of $100 \mathrm{~ms}$. A dedicated software reconstructs the time slices retrieved from all DOMs into a time stream.

\section{Calibration and performances of the line}

\subsection{Darkroom set-up}

After the assembly at NIKHEF, the line has been moved to the CPPM laboratory of Marseille, in a dark room used for the ANTARES detector calibration. The DU1 Calibration consists of several steps: tuning of PMT HV to equalize the PMT gain; absolute and relative time calibration of all PMTs with respect to the master clock; calibration of auxiliary sensors: AHRS, piezos, hydrophone. The procedure followed for DU1, and described in this paper, will be simplified for the next DUs since many steps (e.g. HV tuning and auxiliary sensor calibration) will be performed during PMT and DOM tests and validation to speed up mass production.

The DOMs have been equipped with calibrated acoustic emitters and a laser distribution system for time calibration. The latter consists of a set of 18 optical fibers of equal length and a laser source, synchronized with the detector master clock and triggered by a PPS (pulse per second) signal derived by the master clock system. The fibers point toward one reference PMT of each DOM. This set-up is meant for the inter-DOMs time calibration. The only differences between the dark room setup and the final settings are the absence of the MEOC and junction box and the two 
devices added for calibration. To test the time calibration procedure additional 25 and $50 \mathrm{~km}$ long optical fiber coils have been added between the shore station setup and the DU1 in the dark room. The tests confirm capability of synchronization and syntonization with accuracy well below $1 \mathrm{~ns}$.

\subsection{White rabbit network}

The KM3NeT timing system is based on a customized version of White Rabbit [11]. On Shore a very stable GPS receiver (Symmetricomm XLi), provides the $10 \mathrm{MHz}$ master clock, the pulse per second signal and the NTP absolute time to the whole detector, DAQ and computing system. The GPS is connected to a White Rabbit Switch fabric on shore. The fabric is composed of: a Grand Master switch (WRGMS) which broadcasts Slow Control data from shore to all the CLBs, embedded in the WR precision-time-protocol (ptp); a Level 1 switch (WRL1) which receives detector control data and ptp from the CLB in the base of the DU; a set of $10 \mathrm{Gbps}$ switches that receive the DOM data flow. The KM3NeT time distribution system differs from standard WR since the connection between the master and the slaves is not obtained via point to point connection, but via broadcast. For such reason a custom DAQ and time calibration systems were designed and operated. In the actual KM3NeT design, the CLB on the base uses a "quasi-standard" WR time and communication protocol. That is, its absolute time synchronization and syntonization (known phase delay) between the base and the WRGMS is obtained measuring the Round Trip Time of a timing signal between off-shore and off-shore, after calibrating all the electronics delays and fiber length asymmetries in the upstream/downstream paths. The calibration procedure for the base allowed overall time precision (statistical error) of about $50 \mathrm{ps}$ and accuracy (systematic) of about $500 \mathrm{ps}$, dominated (over long distances) by change of light velocity in fibers due to temperature and pressure effects.

A different approach is used for the DOM CLBs where the absolute time information broadcast by the WRGMS is received by the CLBs after traveling in the upstream fiber network. This time information is used to time-stamp the PMT data offshore. Due to different fiber lengths from DU base to each DOM, a proper time calibration procedure has been carried-out to recover the delay between the base and each DOM, described in section 3.3.

\subsection{PMT performances}

The amplitude calibration of PMTs in the DOMs was achieved through a high voltage scan, around the high voltage value suggested by the vendor for each PMT for a gain of $10^{6}$ in current mode. This value is referred as vendor high voltage $(v H V)$. The procedure aim is at finding the nominal PMT high voltage $(n H V)$ that produces, out of the active base, a signal with a ToT of $26.4 \mathrm{~ns}$ for 1 pe signals in pulsed mode.

The ToT value of a specific PMT corresponds to the mean value of the Gaussian fit to the ToT distribution for this specific PMT. Several examples of ToT distributions and the corresponding Gaussian fits are given in the left plot of figure 2. A scan of the high voltage is performed from $v H V-125 \mathrm{~V}$ up to $v H V+100 \mathrm{~V}$ by step of $25 \mathrm{~V}$. The results of the scan in terms of ToT histogram are shown in the left plot of figure 2 for one PMT of one DOM. The mean ToT is shown as a function of the high voltage variation in the right plot of figure 2 for all PMTs of one DOM. The data points are fitted to a second order polynomial function for the extrapolation to the voltage with a ToT of $26.4 \mathrm{~ns}$. 


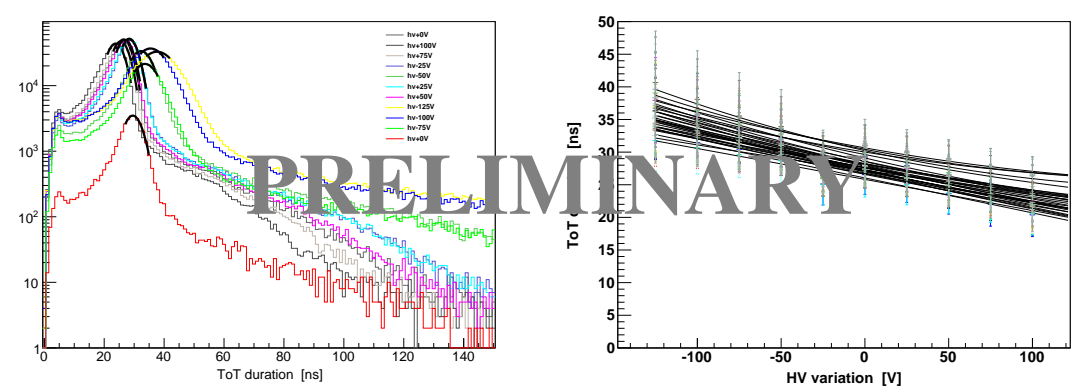

Figure 2: In the left, histograms of the ToT distribution for PMT 8 of DOM 4. Each histogram correspond to a specific high voltage value (from $v H V-125 \mathrm{~V}$ up to $v H V+100 \mathrm{~V}$ by step of $25 \mathrm{~V}$ ). The Gaussian fit to the distribution is also shown. In the right, mean ToT value of the Gaussian fit as a function of the high voltage variation taking the vendor value as a reference for all PMTs of DOM 4. The second order polynomial fit is also shown.

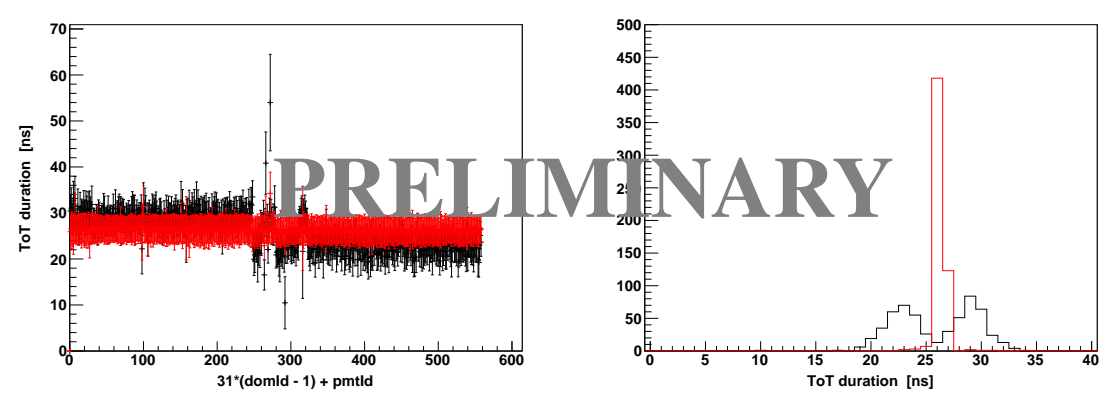

Figure 3: In the left, mean value of the Gaussian fit to the ToT distribution as a function of DOM and PMT identities before (black) and after (red) high voltage adjustment. The error bars correspond to the standard deviation of the Gaussian fit. In the right, histogram of the same ToT values before (black) and after (red) high voltage adjustment.

The values of the ToT is shown in the left plot of figure 3 for the 31 PMTs of the 18 DOMs before (black) and after (red) readjustment in the darkroom. The corresponding histograms are shown in the right plot.

Once the high voltage values have been tuned to nominal $n H V$, the second measurement consists in checking the counting rates. The counting rate for a specific PMT corresponds to the mean value of the Gaussian fit to the distribution of the number of hits per time slice ${ }^{1}$ for this specific PMT. An example of counting rate histogram is given in the left plot of figure 4 for PMT 6 of DOM 4. Each entry of the histogram corresponds to one time slice. The first peak at about $3 \mathrm{kHz}$ is due to the background, i.e. dark count. The second peak is due to the $10 \mathrm{kHz}$ laser emitter and to the background. The mean value of the Gaussian fit to the background histogram is given in the right plot of figure 4 for the 31 PMTs of the 18 DOMs and for a run without the laser emitter. The error bars indicate the standard deviation of the Gaussian fits. 3 PMTs over 558 reached the limit of $20 \mathrm{kHz}$ due to a noisy baseline. Above this limit, the data taking is stopped by the high rate veto (hardware cut) in order to avoid a network congestion. For these specific PMTs the base threshold

\footnotetext{
${ }^{1}$ A time slice corresponds to $100 \mathrm{~ms}$ of data
} 


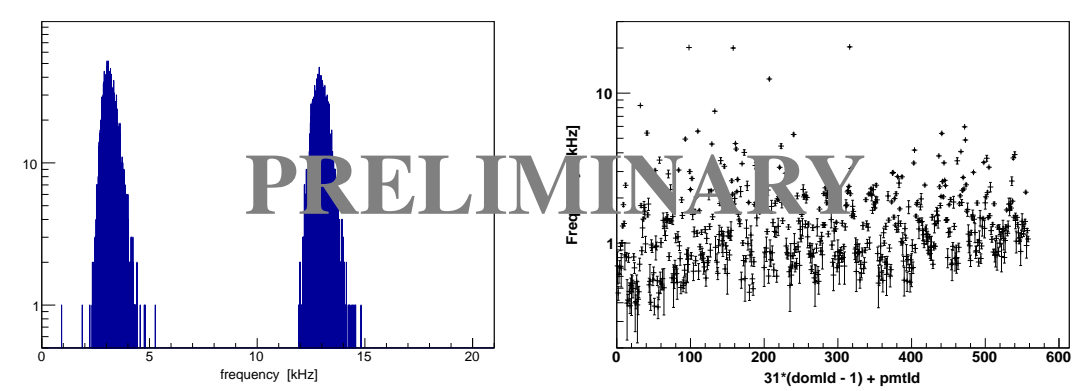

Figure 4: In the left, histogram of the hit frequency (one entry per time slice) for a laser run and for PMT 6 of DOM 4. The first peak corresponds to the dark count and the second to the dark plus laser counts. In the right, mean value of the Gaussian fit to the hit frequency distribution as a function of the DOM and PMT identities. The error bars correspond to the standard deviation of the Gaussian fit.

will be increased. The clusters that could be spotted for the lowest frequencies could be due to the geometrical configuration of the DOMs in their supports.

\subsection{Time calibration}

One of the main points of the darkroom tests is to estimate the accuracy of the time synchronization between the DOMs and to validate the white rabbit scheme. For this purpose, a laser emitter is used. The laser is coupled to 18 optical fibers of the same length which are attached to one reference PMT of each DOM. The laser trigger is synchronized to the PPS of the detector master clock. For each trigger, there is one second of $10 \mathrm{kHz}$ pulses and one second of no activity. Since the 18 PMTs 6 received the laser pulse simultaneously, the time delay due to the clock propagation between the shore station and the DOM CLB can be estimated. The laser effect can be seen in the time difference between consecutive hits, left plot of figure 5, where the peak position reflects the laser period of $10^{5} \mathrm{~ns}$. In the right plot of figure 5, the histogram of the absolute hit time modulo $100 \mu$ s is shown for the PMT 6 of all the DOMs. Since the delay affects the clock signal sent to the CLB of the DOM, the furthest DOM will have the laser pulse earlier compared to the clock signal. Therefore, the most left peak corresponds to DOM 18 and the most right to DOM 1. The mean value of a Gaussian fit to each peak has been used to estimate the clock signal delay of each DOM.

The histogram of the laser peak position is shown for all DOMs together in the right plot of figure 5 . The maximum time difference is below $2 \mathrm{~ns}$. The same operation has been performed after adding $25 \mathrm{~km}$ of optical cable between the shore station and the DOMs. With this cable addition, the clock signal for the DOMs is delayed when compared to the laser pulse. All laser peaks are therefore shifted to earlier times. Since the cable length, folded with the information velocity, is measured by the CLB in the DU base using the WR round trip time measurement, the absolute time for each DOM can always be recovered.

\section{4. conclusion}

With the DU1, the KM3NeT collaboration has started the production phase. The first results obtained in the darkroom are very promising and similar results are expected in the deep sea after 


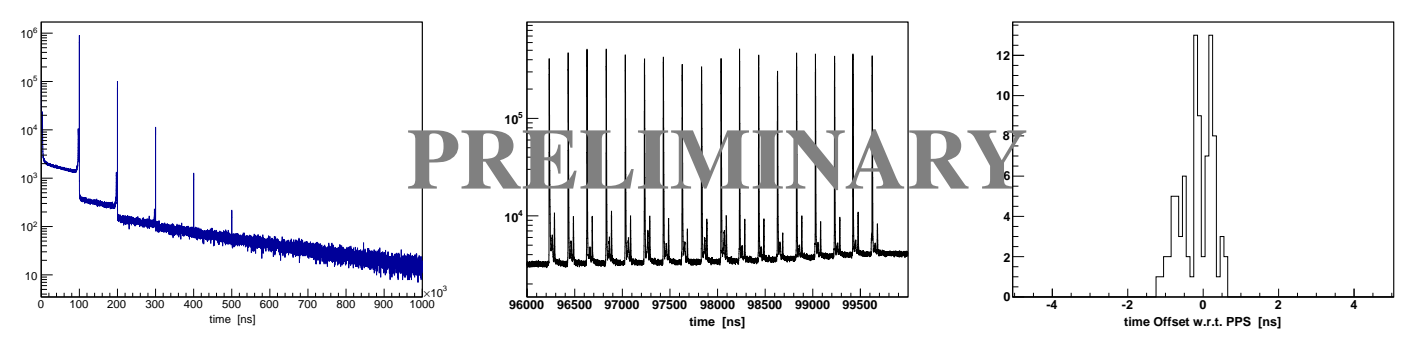

Figure 5: In the left, histogram of the time difference between consecutive hits for the PMT 6 of DOM 4 and for a laser run. In the middle, histogram of the absolute time stamp modulo $100 \mu$ s for PMT 6 of all DOMs. This histogram has been used for estimating the time delay of the clock for each DOM (mean value of a Gaussian fit to each peak). In the right, histogram of the time offset of the PMT 6 of all DOMs with respect to the clock signal after correction of the time offset. 5 runs were considered.

deployment. The tests performed in laboratory have proved that the time synchronization between DOMs of a single line fulfill the requirements with an accuracy of about $1 \mathrm{~ns}$. This accuracy is slightly affected when adding $25 \mathrm{~km}$ of optical cable, nevertheless the absolute DOM time can always be recovered. The tests have also allowed to validate the charge calibration procedure. The PMT response is homogeneous and uniform over all DOMs after HV adjustment.

The next steps for the KM3NeT collaboration are the DU1 deployment expected during summer 2015 and then the completion of the first phase of the Observatory, 31 DUs, expected end of 2016.

\section{References}

[1] KM3NeT Technical Design Report [ISBN 978-90-6488-033-9]

[2] M.G.Aartsen et al. (IceCube Collaboration), Science 342, 947 (2013). doi:10.1126/science.1242856

[3] D.Stranski et al., for the KM3NeT collaboration, proceedings of this conference ID 1108

[4] KM3NeT Collaboration, Eur. Phys. J. C (2014) 74:3056, doi:10.1140/epjc/s10052-014-3056-3

[5] S.Biagi et al., for the KM3NeT collaboration, proceedings of this conference ID 1164

[6] J.A.Aguilar et al., Nuclear Inst. and Methods in Physics Research, A 656 (2011) pp. 11-38 [arXiv:1104.1607v1]

[7] S.Viola et al., for the KM3NeT collaboration, proceedings of this conference ID 1169

[8] P.Kooijman et al., for the KM3NeT collaboration, proceedings of this conference ID 1173

[9] R.Bruijn et al., for the KM3NeT collaboration, proceedings of this conference ID 1157

[10] S.Biagi et al., for the KM3NeT collaboration, proceedings of this conference ID 1172

[11] www.ohwr.org.

[12] V.Kulikovski et al., for the KM3NeT collaboration, proceedings of this conference ID 1170 